\title{
Phase 2 Trial of Pemetrexed in Children and Adolescents with Refractory Solid Tumors: a Children's Oncology Group Study
}

\author{
Anne B. Warwick ${ }^{1}$, Suman Malempati ${ }^{2}$, Mark Krailo ${ }^{3}$, Allen Melemed ${ }^{4}$, Richard Gorlick ${ }^{5}$, \\ Matthew M. Ames ${ }^{6}$, Stephanie L. Safgren ${ }^{6}$, Peter C. Adamson ${ }^{7}$, and Susan M. Blaney ${ }^{8}$ \\ ${ }^{1}$ Uniformed Services University of the Health Sciences, Bethesda, MD \\ ${ }^{2}$ Oregon Health \& Science University, Portland, OR \\ ${ }^{3}$ Keck School of Medicine, University of Southern California, Los Angeles, CA \\ ${ }^{4}$ Eli Lilly and Company, Indianapolis, IN \\ ${ }^{5}$ Montefiore Medical Center, Bronx, NY \\ ${ }^{6}$ Department of Molecular Pharmacology and Experimental Therapeutics, Mayo Clinic, \\ Rochester, MN \\ ${ }^{7}$ Children's Hospital of Philadelphia, Philadelphia, PA \\ ${ }^{8}$ Texas Children's Cancer Center, Baylor College of Medicine, Houston, TX
}

\section{Abstract}

Background-Pemetrexed is a multi-targeted antifolate that inhibits key enzymes involved in nucleotide biosynthesis. We performed a phase 2 trial of pemetrexed in children with refractory or recurrent solid tumors, including CNS tumors, to estimate the response rate and further define its toxicity profile.

Procedure-Pemetrexed, at a dose of $1910 \mathrm{mg} / \mathrm{m}^{2}$, was administered as a 10-minute intravenous infusion every 21 days. Patients also received vitamin $\mathrm{B}_{12}$, daily multivitamin supplementation, and dexamethasone. A two-stage design $(10+10)$ was employed in each of the following disease strata: osteosarcoma, Ewing sarcoma/peripheral primitive neuroectodermal tumor (PNET), rhabdomyosarcoma, neuroblastoma, ependymoma, medulloblastoma/supratentorial PNET, and non-brainstem high-grade glioma.

Results-Seventy-two eligible subjects (39 males) were enrolled. Median age was 11 years (range 3-23). Sixty-eight were evaluable for response. The median number of cycles administered was 2 (range 1-13). No complete or partial responses were observed. Stable disease, for a median of 5 (range 4-13) cycles, was observed in 5 patients (ependymoma, Ewing sarcoma, medulloblastoma, neuroblastoma, osteosarcoma; $n=1$ each). Neutropenia (44\%), anemia (35\%), and elevated alanine transaminase (35\%) attributable to pemetrexed were the most commonly recurring toxicities observed in patients receiving multiple cycles. Other toxicities attributed to pemetrexed occurring in $\geq 10 \%$ of cycles included thrombocytopenia (30\%), fatigue (18\%), nausea (14), hyperglycemia (13\%), rash (11\%), vomiting (13\%), and hypophosphatemia (11\%).

Conclusions-Pemetrexed, administered as an intravenous infusion every 21 days, was tolerable in children and adolescents with refractory solid tumors, including CNS tumors, but did not show evidence of objective anti-tumor activity in the childhood tumors studied.

Corresponding Author: USU, Department of Pediatrics, 4301 Jones Bridge Road, Bethesda, MD 20814, anne.warwick@ usuhs.mil. Presented in part at the 46th Annual Meeting of the American Society of Clinical Oncology, Chicago, IL, June 2010. 


\section{Keywords}

pemetrexed; phase 2; antifolate

\section{INTRODUCTION}

Pemetrexed (LY231514; ALIMTA®; pemetrexed disodium; Eli Lilly and Company, Indianapolis, Indiana), is a novel folate antimetabolite that inhibits several folate-dependent enzymes, including thymidylate synthase (TS), dihydrofolate reductase (DHFR), glycinamide ribonucleotide formyl transferase (GARFT)[1], and to a lesser degree, aminoimidazole carboxamide formyl transferase [2]. Pemetrexed primarily enters cells through the reduced folate carrier and to a lesser extent, through membrane folate-binding transport systems [3]. Following cell entry, pemetrexed is polyglutamated by folylpolyglutamate synthetase [4], resulting in increased cellular retention of the molecule, which leads to a longer exposure and increased intracellular concentration $[2,3]$ as well as a significant increase in affinity for TS and GARFT [2-7].

Folate antagonists have been used in the treatment of childhood cancers since the 1940s when aminopterin was introduced; its use was superseded by methotrexate, an antifol with a better therapeutic index and a more reliable level/dose relationship [8]. Methotrexate is now widely used in the treatment of childhood malignancies including leukemias, lymphomas, and osteosarcoma. There is also rationale for the use of antifols in primary CNS tumors such as ependymoma, which have been shown to have high expression of folate receptors. Because observational and preclinical data suggest that folates also play a role in neuroblastoma metabolism and occurrence [9-12], the use of antifol chemotherapy may have a role in its treatment as well. Fluorouracil is another antifol currently used in pediatric cancer for treatment of hepatoblastoma and nasopharyngeal carcinoma. Resistance to the antifols currently used in the clinic, occurring as a result of alterations in folate transport as well as up-regulation of DHFR or down-regulation of folylpolyglutamate synthetase, has led to the development of new antifols such as edatrexate, raltitrexed, trimetrexate, as well as pemetrexed [8].

Pemetrexed was approved by the United States Food and Drug Administration as first-line therapy for the treatment of malignant mesothelioma or as second-line treatment in combination with cisplatin for patients with locally advanced or metastatic nonsquamous non-small cell lung cancer. A phase 1 study of pemetrexed in children and adolescents with recurrent solid tumors, including CNS tumors, was performed by the Children's Oncology Group (COG) defined the maximum-tolerated dose (MTD) as $1910 \mathrm{mg} / \mathrm{m}^{2}$ for children, with a toxicity profile similar to that observed in adults [13].

This report describes the results of a COG single-agent phase 2 trial of pemetrexed, administered every 21 days with folic acid and vitamin $\mathrm{B}_{12}$ supplementation, that was designed to determine the response rate in children with recurrent or refractory osteosarcoma, Ewing sarcoma/peripheral primitive neuroectodermal tumor (PNET), rhabdomyosarcoma, neuroblastoma, ependymoma, medulloblastoma/supratentorial PNET, or non-brainstem high-grade glioma. Secondary aims included further assessment of pemetrexed toxicities in children, as well as an exploration of a potential correlation between the presence of a polymorphism in the TS gene and/or gene promoter and pemetrexed toxicity. 


\section{MATERIALS AND METHODS}

\section{Patient Eligibility}

Patients between the ages of 6 months and 21 years of age at time of original diagnosis of a recurrent or refractory osteosarcoma, Ewing sarcoma/peripheral PNET, rhabdomyosarcoma, neuroblastoma, ependymoma, medulloblastoma/supratentorial PNET, or non-brainstem high-grade glioma that was measureable by the Response Evaluation Criteria In Solid Tumors (RECIST) [14] with the exception of neuroblastoma, were eligible. Patients with neuroblastoma were enrolled into two strata: patients with disease measurable by RECIST and patients with disease noted on an iodine meta-iodobenzylguanidine (MIBG) scan only. All patients were required to have recovered from the toxic effects of prior therapy and have a Karnofsky ( $>16$ years) or a Lansky ( $₫ 6$ years) performance score of 250 . Organ function requirements included a bilirubin $\leq .5$ times the upper limit of normal, alanine aminotransferase (ALT) $\leq 10 \mathrm{U} / \mathrm{L}$, serum albumin $\geq 2 \mathrm{~g} / \mathrm{dL}$, and an age-adjusted normal serum creatinine or a creatinine clearance $\geq 70 \mathrm{~mL} / \mathrm{min} / 1.73 \mathrm{~m}^{2}$. For patients without bone marrow involvement, an absolute neutrophil count (ANC) $\geq 1000 / \mathrm{mcL}$, a transfusionindependent platelet count $\geq 100,000 / \mathrm{mcL}$, and a hemoglobin $\geq 8 \mathrm{~g} / \mathrm{dL}$ were required. Patients with known bone marrow metastatic disease were eligible for the study but were not evaluable for hematologic toxicity.

Intervals from prior therapy to enrollment included 3 weeks for conventional chemotherapy (4 if nitrosourea had been administered); 1 week for a biologic agent; 2 weeks for local palliative radiotherapy (XRT); 6 weeks if substantial bone marrow radiation such as prior craniospinal XRT or $>50 \%$ radiation of the pelvis; and 6 months from a stem cell transplant.

Patients were excluded if they were pregnant or breast-feeding; were receiving another investigational drug or other anti-cancer agent; or had a history of a prior allergic reaction to mannitol, an uncontrolled infection, a pleural effusion, ascites, or prior treatment with pemetrexed. In addition, patients must not have received myeloid growth factors within the week prior to study entry ( 2 weeks if pegfilgrastim had been given), any drug excreted via the renal tubules for at least 2 days pre- and post-pemetrexed, or any corticosteroid, unless patients had a CNS tumor and were on a stable or decreasing dose for at least 7 days prior to study entry.

This study was approved by individual institutional review boards (IRBs), as well as the National Cancer Institute Pediatric Central IRB. All patients or their parents/legal guardians signed a document of informed consent; assent was obtained as appropriate, according to individual institutional guidelines.

\section{Treatment Plan}

Pemetrexed $1910 \mathrm{mg} / \mathrm{m}^{2}$ (or $60 \mathrm{mg} / \mathrm{kg}$ for patients <12 months old), maximum dose 3820 $\mathrm{mg}$, was administered as a 10-minute intravenous infusion once every 21 days. All patients were required to take a daily oral multivitamin supplement containing at least $400 \mu \mathrm{g}$ of folic acid for at least 5 of the 7 days prior to the first pemetrexed dose and for at least 21 days after the last dose. Intramuscular injections of cyanocobalamin (vitamin $\mathrm{B}_{12}, 500$ to $1000 \mu \mathrm{g}$ based on age) were administered before the first dose of pemetrexed and after every third course. With each treatment course, oral dexamethasone, $0.1 \mathrm{mg} / \mathrm{kg} / \mathrm{dose}$ (maximum, $4 \mathrm{mg} /$ dose) was administered twice daily for 3 days, beginning 1 day before the pemetrexed infusion. A 5-HT3 receptor antagonist was also permitted 30 minutes prior to pemetrexed according to institutional guidelines. 
Cycles were repeated every 21 days if the patient did not have progressive disease and had recovered from the prior course with an ANC $\geq 1000 / \mathrm{mcL}$ and platelets $\geq 100,000 / \mathrm{mcL}$ and met all the other organ function requirements as required at study entry.

Adverse events were graded according to the National Cancer Institute Common Terminology Criteria (CTCAE), version 3.0 [15].

Reduction of the pemetrexed dose to $1470 \mathrm{mg} / \mathrm{m}^{2}$ ( $45 \mathrm{mg} / \mathrm{kg}$ for children $<12$ months) was made for isolated grade 4 neutropenia of $>7$ days' duration that recurred despite filgrastim support, grade 4 thrombocytopenia $>7$ days' duration, grade 3 or 4 thrombocytopenia requiring transfusion more than twice per cycle, myelosuppression that caused a delay of $>14$ days between cycles, or grade 3 or 4 non-hematologic toxicity that returned to baseline (eligibility) levels by 14 days after the planned start of the next treatment cycle. Exceptions to the non-hematologic toxicity resulting in dose reduction included grade 3 or 4 nausea or vomiting, grade 3 hepatic aminotransferase elevations that returned to grade 1 prior to the next cycle, grade 3 fever or infection, or grade 3 mucositis. Leucovorin rescue, $100 \mathrm{mg} / \mathrm{m}^{2}$ intravenous loading dose followed by $50 \mathrm{mg} / \mathrm{m}^{2}$ orally or intravenously every 6 hours (up to 8 days), was initiated for grade 4 mucositis.

Patients who had dose-limiting toxicity that did not resolve to criteria that met eligibility requirements by 14 days after the planned start of the next treatment cycle or those who required a second dose reduction were removed from protocol therapy.

Patient Evaluations-Patient history, including performance status, was obtained before study entry and with each treatment course. Physical examination was performed and laboratory studies were obtained before treatment, weekly throughout the first course of treatment, and before each subsequent course. CBCs were obtained at least twice weekly during the first course and weekly thereafter. In consenting patients, blood for pharmacogenetic studies was drawn once at study entry. Patients with measurable disease were evaluated using RECIST.(14) Patients with neuroblastoma noted only by an MIBG scan were evaluated by central review using the Curie scale [16].

Study Design and Response Evaluation-A two-stage design was used to evaluate pemetrexed in seven disease strata: osteosarcoma, Ewing sarcoma/peripheral PNET, rhabdomyosarcoma, neuroblastoma, ependymoma, medulloblastoma/supratentorial PNET, and non-brainstem high-grade glioma. At the first stage for each stratum, 10 patients were enrolled. If no patient experienced an objective response, pemetrexed was considered inactive in that stratum, and enrollment to that stratum was terminated. If $\geq 1$ patient achieved a partial response or complete response, 10 additional patients would be enrolled to that stratum. Pemetrexed would be considered to be active if $\geq 3$ of 20 patients in the expanded stratum experienced a partial or complete response. With this design, pemetrexed would be identified as inactive if the true response rate was $5 \%$ with a probability of 0.93 , and would be identified as active if the true response rate was $30 \%$ with a probability of 0.95 .

Any eligible patient who received at least one dose of pemetrexed was considered evaluable for response provided: (1) the patient demonstrated progressive disease or died while on protocol therapy; or (2) the patient was observed on protocol therapy for at least one cycle and the tumor was not removed surgically prior to the time a complete or partial response was confirmed; or (3) the patient demonstrated a complete or partial response as confirmed by central review of radiographic images. The evaluation period for determination of the overall best response was six treatment cycles. All other patients were considered nonresponders. 
Toxicity Evaluation-Each cycle in which pemetrexed was administered to an eligible patient was considered in the analysis of toxicity. The treating physician assigned an attribution for each CTC-gradable adverse event as unrelated, unlikely, possibly, probably, or definitely related to pemetrexed. The relative frequency of each adverse event considered possibly, probably, or likely related to pemetrexed was estimated as the proportion of all toxicity-evaluable cycles in which such a toxicity was observed.

The probability that a particular adverse experience of grade 1 or greater that occurred in a toxicity-evaluable treatment cycle was related to a specific TS promoter was evaluated using logistic regression analysis. The model included a term to quantify cumulative dose of pemetrexed received and a categorical variable quantifying the alleles observed in the MTHFR gene. Attribution to pemetrexed was not considered for this analysis. Only the adverse experiences that occurred in $10 \%$ or more of cycles were considered in the analysis because the power to detect associations with less frequently occurring adverse experiences was low.

Pharmacogenetic Studies-DNA was extracted from the buffy coat of a pre-treatment blood sample collected in a 10-mL (purple-topped) EDTA tube. Standard procedures for DNA isolation using a QIAGEN kit (Valencia, CA) were followed. Three variations in the TS gene (TYMS) were analyzed by two PCR amplifications with sequence-specific primers for the regions of interest. The amplified DNA was sequenced in the Mayo Advanced Genomics Technology Center (AGTC) sequencing core laboratory using an ABI PRISM® 3730xl DNA analyzer (Life Technologies Corporation, Carlsbad, CA). Similarly, two regions containing a single nucleotide polymorphism (SNP) in the methylenetetrahydrofolate reductase (MTHFR) gene were amplified with specific primers and sequenced in the Mayo AGTC core lab. All methods were validated using a set of 60 DNA samples from Caucasian individuals obtained from the Coriell Institute (Camden, New Jersey). Allele frequencies were comparable with published data in the US National Center for Biotechnology Information [17]. The DNA primer sequences are listed in Table I. Primers for MTHFR were designed in the laboratory of Matthew Ames, PhD (Mayo Clinic, Rochester MN). Primers for the $3^{\prime}$ untranslated region and the 5' Thymidylate Synthetase Enhancer Region (TSER) of TYMS were from Ulrich et al. and Kawakami et al., respectively $[18,19]$.

\section{RESULTS}

\section{Patient Characteristics}

This study (study code: ADVL0525; ClinicalTrials.gov identifier: NCT00520936) was opened in September 2007 and closed in October 2009. Data as of March 2010 were used in the analyses. Seventy-five patients enrolled, of whom 72 were eligible (Table II). Three patients were ineligible: two because protocol therapy was started prior to enrollment (administration of multivitamin in one case, dexamethasone in the other); and because one patient did not have a RECIST-measurable disease. Seventy of the eligible patients were fully evaluable for toxicity. Two patients did not start protocol therapy because of a rapidly progressive tumor. Sixty-eight patients were evaluable for response. One had baseline scans that were > 2 weeks old at study entry; and one patient had therapy stopped 5 minutes into the first dose because of an allergic reaction (no follow-up scans were obtained).

\section{Tumor Response}

Of the 68 patients evaluable for response, there were 10 patients in each disease stratum with the exception of the rhabdomyosarcoma stratum, which included only 8 evaluable patients. No partial or complete responses were observed. The median number of treatment 
cycles was 2 (range 1-13). Thirteen patients received $\geq 3$ cycles. Stable disease occurred in five patients across a spectrum of solid tumors, including one patient each with ependymoma (5 cycles), Ewing sarcoma (13 cycles), medulloblastoma (4 cycles), neuroblastoma ( 5 cycles), and osteosarcoma ( 5 cycles). These patients received a median of 5 (range 4-13) cycles of therapy. The accrual was halted after the first stage in all disease strata because the target response rate was not met.

\section{Toxicity}

The 70 patients who were fully evaluable for toxicity received 112 treatment cycles. Two allergic reactions occurred, each with the first dose of the drug; both patients discontinued study participation. Two patients had a prolonged elevation of ALT in cycle 1. One patient discontinued protocol therapy; the other received reduced-dose pemetrexed per protocol guidelines for the remaining time on study.

Toxicities possibly, probably, or definitely related to pemetrexed that occurred in more than $10 \%$ of all treatment cycles are listed by grade in Table III. Hematologic toxicity was the most common, with neutropenia occurring in $44 \%$ of all cycles, anemia in $35 \%$, lymphopenia in $23 \%$, and thrombocytopenia in $30 \%$. Transaminase elevation was next most likely to occur: elevations in ALT occurred in 35\% and aspartate aminotransferase, in 33 $(33 \%)$ of all cycles. Other toxicities included fatigue $(18 \%)$, rash $(11 \%)$, nausea $(14 \%)$, vomiting (13\%), hyperglycemia (13\%), and hypophosphatemia (11\%).

\section{Pharmacogenetic Studies}

DNA from 62 patients was analyzed for the presence of genetic variations in the TS and MTHFR genes, looking specifically for variable numbers of tandem repeats, deletions, or SNPs (Table I).

Four patients exhibited genetic changes of the TS gene (i.e., had three copies of the repeat region on both alleles with $\mathrm{G}$ at the SNP site), which have been associated with increased response to antifol therapy [20]. There was no significant difference in response to therapy between these four and the other 58 patients.

Four patients were homozygous and 30 heterozygous for a 6bp deletion $447 \mathrm{bp}$ downstream of the TS transcription stop codon, which has been suggested to alter the TS mRNA stability.(20) Five patients were homozygous and 27 heterozygous for a variant allele at amino acid position $222 \mathrm{Val}$ of the MTHFR gene, which is associated with an elevated homocysteine levels under conditions of impaired folate status [21], and thus might be expected to result increased toxicity with pemetrexed. Four patients were homozygous and 23 heterozygous for a variant allele at amino acid position 429Ala of the MTHFR gene, which is associated with decreased MTHFR activity [22]. There were no significant associations between these genotypes and any grade 3 or higher toxicities attributed to pemetrexed.

\section{DISCUSSION}

This trial confirmed the tolerability of pemetrexed at the MTD $\left(1910 \mathrm{mg} / \mathrm{m}^{2}\right)$ established in the pediatric phase 1 trial [13]. In that trial, vitamin supplementation with $\mathrm{B}_{12}$ and folic acid (along with dexamethasone for prevention of skin rash) allowed escalation of the dose to a considerably higher level than the MTD of 500 to $600 \mathrm{mg} / \mathrm{m}^{2}$ found in the initial adult phase 1 trials [23, 24] With the addition ofvitamin supplementation, subsequent trials in adults demonstrated improved tolerability of pemetrexed without decreased efficacy [25, 26], and pemetrexed doses have been successfully escalated up to $1200 \mathrm{mg} / \mathrm{m}^{2}$ in adult phase 1 studies [27]. Consistent with prior adult and pediatric trials, the most common toxicities in 
this phase 2 study were hematologic. We found no association between the MTHFR genotype and risk for toxicity.

In this study, we evaluated activity in a variety of pediatric solid tumors (osteosarcoma, Ewing sarcoma/peripheral PNET, rhabdomyosarcoma, neuroblastoma, ependymoma, medulloblastoma/supratentorial PNET, or non-brainstem high-grade glioma), but found no objective anti-tumor activity. Because osteosarcoma cells are sensitive to antifolate therapy, and methotrexate is a standard component of osteosarcoma therapy, one would have expected more robust activity from the more potent antifolate agent pemetrexed. The reason for lack of single-agent activity in osteosarcoma is unclear. Although pemetrexed has shown in vitro activity against tumor cells that are resistant to other antifols [28, 29], it is possible that cross-resistance to pemetrexed was present in this patient population that was likely heavily pre-treated with methotrexate. In addition, whereas folate and $\mathrm{B}_{12}$ supplementation have not adversely impacted efficacy in NSCLC [30], we cannot completely exclude a negative impact of vitamin supplementation on pemetrexed activity in osteosarcoma.

Lack of single-agent activity does not necessarily preclude further development of novel anti-neoplastic agents if there is evidence of synergy with other agents. There is evidence of enhanced anti-tumor activity of pemetrexed in combination with cisplatin and gemcitabine for NSCLC and mesothelioma. However, at this time, there is no clear evidence to suggest synergistic activity of pemetrexed with other agents in osteosarcoma or other pediatric malignancies. In addition, whereas pemetrexed is reasonably well tolerated as a single agent, overlapping toxicity with standard myelosuppressive chemotherapy will make combination strategies difficult.

In conclusion, pemetrexed shows little single-agent activity in pediatric solid tumors, and data from this study do not support further development of this drug for these diseases.

\section{Acknowledgments}

Financial Support: Eli Lilly and Company and National Cancer Institute Grant numbers: U10 CA98543 and U10 CA98413

\section{References}

1. Jones RJ, Twelves CJ. Pemetrexed: A multitargeted antifolate (alimta, ly-231514). Expert Rev Anticancer Ther. 2002; 2:13-22. [PubMed: 12113062]

2. Paz-Ares L, Bezares S, Tabernero JM, et al. Review of a promising new agent--pemetrexed disodium. Cancer. 2003; 97:2056-2063. [PubMed: 12673697]

3. Hazarika M, White RM, Johnson JR, et al. Fda drug approval summaries: Pemetrexed (alimta). Oncologist. 2004; 9:482-488. [PubMed: 15477632]

4. Shih C, Chen VJ, Gossett LS, et al. Ly231514, a pyrrolo[2,3-d]pyrimidine-based antifolate that inhibitsmultiple folate-requiring enzymes. Cancer Res. 1997; 57:1116-1123. [PubMed: 9067281]

5. Mendelsohn LG, Shih C, Chen VJ, et al. Enzyme inhibition, polyglutamation, and the effect of ly231514 (mta) on purine biosynthesis. Semin Oncol. 1999; 26:42-47. [PubMed: 10598554]

6. Schultz RM, Patel VF, Worzalla JF, et al. Role of thymidylate synthase in the antitumor activity of the multitargeted antifolate, ly231514. Anticancer Res. 1999; 19:437-443. [PubMed: 10226579]

7. Shih C, Habeck LL, Mendelsohn LG, et al. Multiple folate enzyme inhibition: Mechanism of a novel pyrrolopyrimidine-based antifolate ly231514 (mta). Adv Enzyme Regul. 1998; 38:135-152. [PubMed: 9762351]

8. Hum MC, Kamen BA. Folate, antifolates, and folate analogs in pediatric oncology. Invest New Drugs. 1996; 14:101-111. [PubMed: 8880397]

9. Ahmad K. Folic acid in food prevents neuroblastoma. Lancet Oncol. 2003; 4:649. [PubMed: 14661642] 
10. Field MS, Anguera MC, Page R, et al. 5,10-methenyltetrahydrofolate synthetase activity is increased in tumors and modifies the efficacy of antipurine ly309887. Arch Biochem Biophys. 2009; 481:145-150. [PubMed: 19022216]

11. Field MS, Szebenyi DM, Stover PJ. Regulation of de novo purine biosynthesis by methenyltetrahydrofolate synthetase in neuroblastoma. J Biol Chem. 2006; 281:4215-4221. [PubMed: 16365037]

12. French AE, Grant R, Weitzman S, et al. Folic acid food fortification is associated with a decline in neuroblastoma. Clin Pharmacol Ther. 2003; 74:288-294. [PubMed: 12966372]

13. Malempati S, Nicholson HS, Reid JM, et al. Phase i trial and pharmacokinetic study of pemetrexed in children with refractory solid tumors: The children's oncology group. J Clin Oncol. 2007; 25:1505-1511. [PubMed: 17442992]

14. Therasse P, Arbuck SG, Eisenhauer EA, et al. New guidelines to evaluate the response to treatment in solid tumors. European organization for research and treatment of cancer, national cancer institute of the united states, national cancer institute of canada. J Natl Cancer Inst. 2000; 92:205216. [PubMed: 10655437]

15. CTEP. CTEP. Common terminology criteria for adverse events, version 3.0. 2006

16. Ady N, Zucker JM, Asselain B, et al. A new 123i-mibg whole body scan scoring method-application to the prediction of the response of metastases to induction chemotherapy in stage iv neuroblastoma. Eur J Cancer. 1995; 31A:256-261. [PubMed: 7718334]

17. (dbSNP). DoSNP. Bethesda (md): National center for biotechnology information, national library of medicine; Dbsnp accession: \{rs1801133, rs1801131, rs34743033, rs34489327\}. Available fromURL: http://www.Ncbi.Nlm.Nih.Gov/snp/

18. Ulrich CM, Curtin K, Potter JD, et al. Polymorphisms in the reduced folate carrier, thymidylate synthase, or methionine synthase and risk of colon cancer. Cancer Epidemiol Biomarkers Prev. 2005; 14:2509-2516. [PubMed: 16284371]

19. Kawakami K, Omura K, Kanehira E, et al. Polymorphic tandem repeats in the thymidylate synthase gene is associated with its protein expression in human gastrointestinal cancers. Anticancer Res. 1999; 19:3249-3252. [PubMed: 10652619]

20. Pharmacogenomics Knowledge Base (PharmGKB). [Last accessed November 1, 2011] Variant at chr18:647646. Available from URL: http://www.pharmgkb.org/views/reports/ loadVariantReport.action?varRptId=127844061\&tabType=17-tabview=tab2

21. PharmGKB. [Last accessed November 1, 2011] Variant rs1801133 at chr1:11778965 in clcn6/ mthfr. Available from URL: http://www.pharmgkb.org/search/search.action? exactMatch=true \&query $=$ Variant+rs1801133+at+chr1\%3A11778965+in + CLCN6\%2FMTHFR\&autoFlag=false

22. [Last accessed November 23, 2011] 5,10-Methylenetetrahydrofolate Reductase; MTHFR, at Online Mendelian Inheritance in Man. Available from URL: http://omim.org/entry/ 607093\#607093_AllelicVariant0004

23. Fossella FV, Gatzemeier U. Phase i trials of pemetrexed. Semin Oncol. 2002; 29:8-16. [PubMed: 12023787]

24. Rinaldi DA, Kuhn JG, Burris HA, et al. A phase i evaluation of multitargeted antifolate (mta, ly231514), administered every 21 days, utilizing the modified continual reassessment method for dose escalation. Cancer Chemother Pharmacol. 1999; 44:372-380. [PubMed: 10501910]

25. Adjei AA. Pemetrexed (alimta), a novel multitargeted antineoplastic agent. Clin Cancer Res. 2004; 10:4276s-4280s. [PubMed: 15217974]

26. Scagliotti GV, Shin DM, Kindler HL, et al. Phase ii study of pemetrexed with andwithout folic acid and vitamin b12 as front-line therapy in malignant pleural mesothelioma. J Clin Oncol. 2003; 21:1556-1561. [PubMed: 12697881]

27. Nakagawa K, Kudoh S, Matsui K, et al. A phase i study of pemetrexed (ly231514) supplemented with folate and vitamin b12 in japanese patients with solid tumours. Br J Cancer. 2006; 95:677682. [PubMed: 16940981]

28. Zhao R, Babani S, Gao F, et al. The mechanism of transport of the multitargeted antifolate (mta) and its cross-resistance pattern in cells with markedly impaired transport of methotrexate. Clin Cancer Res. 2000; 6:3687-3695. [PubMed: 10999762] 
29. Schultz RM, Chen VJ, Bewley JR, et al. Biological activity of the multitargeted antifolate, mta (ly231514), in human cell lines with different resistance mechanisms to antifolate drugs. Semin Oncol. 1999; 26:68-73. [PubMed: 10598558]

30. Hanna N, Shepherd FA, Fossella FV, et al. Randomized phase iii trial of pemetrexed versus docetaxel in patients with non-small-cell lung cancer previously treated with chemotherapy. J Clin Oncol. 2004; 22:1589-1597. [PubMed: 15117980] 


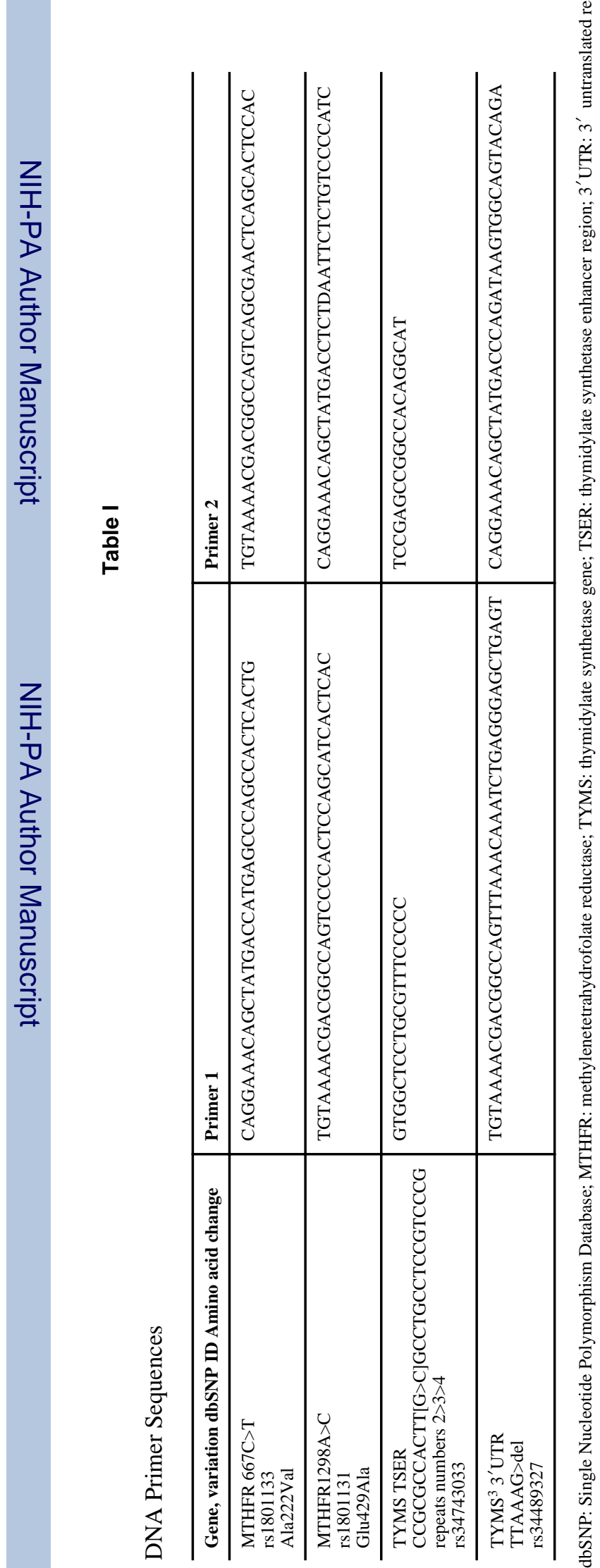


Table II

Baseline Characteristics of 72 Eligible Patients

\begin{tabular}{lc}
\hline Gender, n, male:female & $39: 33$ \\
\hline Median age, years (range) & $11(3-23)$ \\
\hline Diagnoses, $\mathbf{n}$ & Eligible (Evaluable) \\
\hline All patients & $72(68)$ \\
Osteosarcoma & $10(10)$ \\
Ewing/PNET & $10(10)$ \\
Rhabdomyosarcoma & $9(8)$ \\
Neuroblastoma & $11(10)$ \\
Ependymoma & $10(10)$ \\
Medulloblastoma & $10(10)$ \\
Non-brainstem high-grade glioma & $12(10)$ \\
\hline Race, $\mathbf{n}$ (\%) & $\mathrm{n}(\%)$ \\
\hline Caucasian & $46(64)$ \\
Black & $15(21)$ \\
American Indian, Aleutian, Eskimo & $2(3)$ \\
Chinese & $1(1)$ \\
\hline Osian Indian, Pakistani & $1(1)$ \\
\hline Onknown & $4(6)$ \\
\hline
\end{tabular}

PNET, primitive neuroectodermal tumor. 


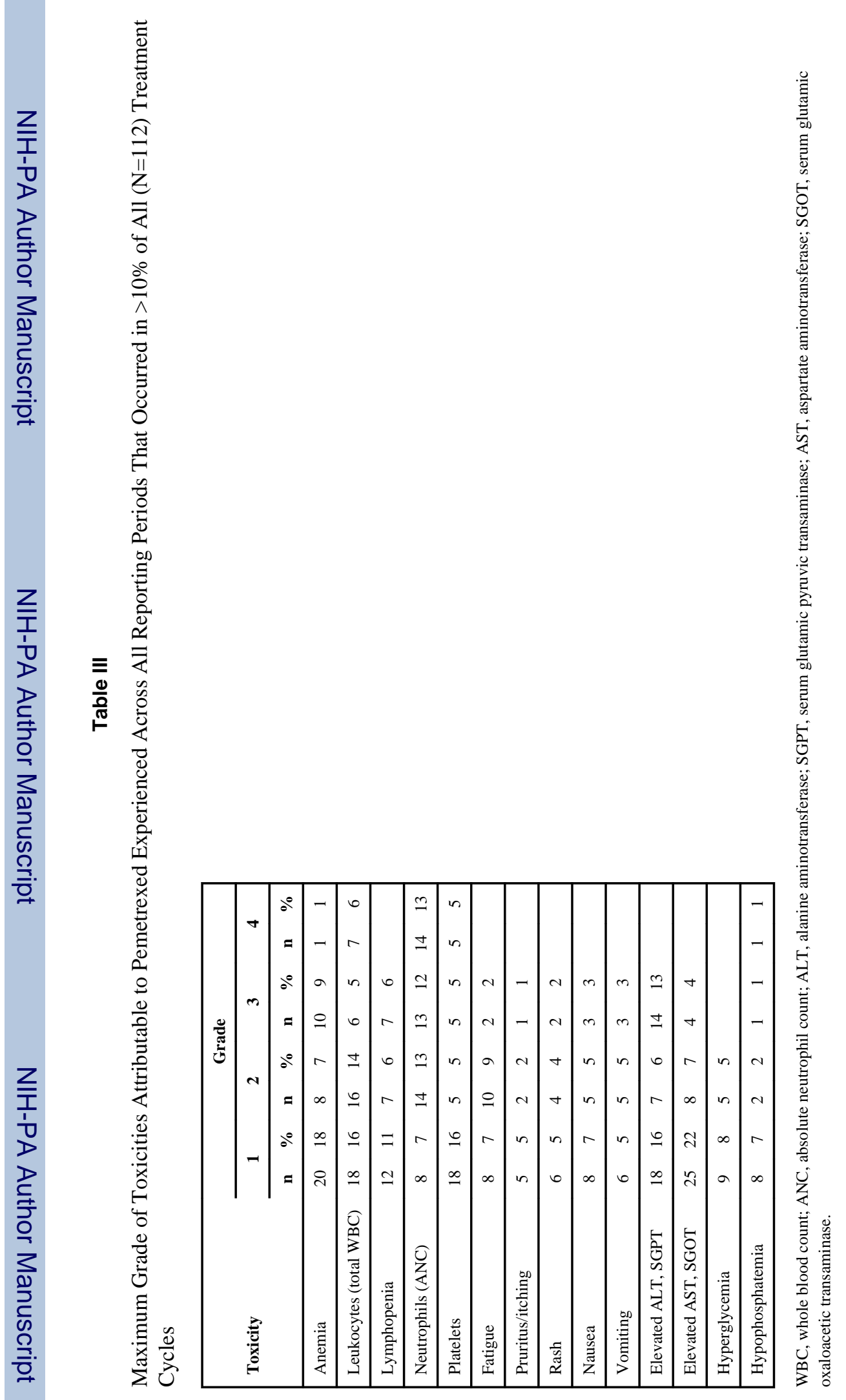

Pediatr Blood Cancer. Author manuscript; available in PMC 2014 February 01. 